\title{
Simulation and optimization of tuneable microstrip patch antenna for fifth-generation applications based on graphene
}

\author{
Hamzah M. Marhoon ${ }^{1}$, Nidal Qasem² \\ ${ }^{1,2}$ Department of Electronics and Communications Engineering, Al-Ahliyya Amman University, Jordan \\ ${ }^{1}$ Department of Computer Techniques Engineering, Al-Esraa University College, Iraq
}

\begin{tabular}{l}
\hline Article Info \\
\hline Article history: \\
Received Oct 27, 2019 \\
Revised Feb 29, 2020 \\
Accepted Apr 29, 2020 \\
\hline
\end{tabular}

Keywords:

$5 \mathrm{G}$

$60 \mathrm{GHz}$

Graphene

Microstrip patch antenna

Tuneable antenna

\begin{abstract}
Microstrip patch antennas (MPAs) are known largely for their versatility in terms of feasible geometries, making them applicable in many distinct circumstances. In this paper, a graphene-based tuneable single/array rectangular microstrip patch antenna (MPA) utilizing an inset feed technique designed to function in multiple frequency bands are used in a fifth-generation $(5 \mathrm{G})$ wireless communications system. The tuneable antenna is used to eliminate the difficulties caused by the narrow bandwidths typically associated with MPAs. The graphene material has a reconfigurable surface conductivity that can be adjusted to function at the required value, thus allowing the required resonance frequency to be selected. The simulated tuneable antenna comprises a copper radiating patch with four graphene strips used for tuning purposes and is designed to cover a wide frequency band. The proposed antenna can be tuned directly by applying a direct current (DC) voltage to the graphene strips, resulting in a variation in the surface impedance of the graphene strips and leading to shifts in the resonance frequency.
\end{abstract}

Copyright $(2020$ Institute of Advanced Engineering and Science. All rights reserved.

\section{Corresponding Author:}

Nidal Qasem,

Department of Electronics and Communications Engineering, Faculty of Engineering,

Al-Ahliyya Amman University,

Zip-code (Postal Address): 19328, Amman, Jordan.

Email: Ne.qasem@ammanu.edu.jo

\section{INTRODUCTION}

Wireless communication technology has developed rapidly to meet the demand for high traffic capacities in electronic devices. Fifth-generation (5G) technology utilizes higher-frequency bands in order to provide the large information capacities needed to support multi-Gbps information rates and collect infinite information broadcasts using the latest mobile technology $[1,2]$.

Millimetre-wave (mm-wave) (30 to $300 \mathrm{GHz}$ ) technology is currently receiving increased attention due to increasing demands for ultra-broadband communication equipment in wireless communications. The main problem in mm-wave communication systems is to design a small-sized antenna with a wide-band feature that includes the entire accessible band. In addition, millimetre electronic embedded circuits integrated with mm-wave antennas are physically compact and technologically efficient. In the mm-wave range, using the unlicensed frequency band from 57 to $64 \mathrm{GHz}$ ( $7 \mathrm{GHz}$ bandwidth) that relies on the V-band (50 to $70 \mathrm{GHz}$ ) for industrial, scientific and medical applications shows promise [3, 4].

This huge quantity of spectral space $(\approx 7 \mathrm{GHz})$ around the $60 \mathrm{GHz}$ frequency provides room for the ultra- high-speed transfer of large quantities of information from short-range applications, such as high-definition video transmissions and point-to-point desktop connections. The next generation of wireless technologies is expected to face spectrum scarcity in the frequency band below $10 \mathrm{GHz}$, due to the exploding number of products, and data-intensive applications running on today's consumer electronic products. 
Hence, wireless communications at $60 \mathrm{GHz}$ have attracted growing interest for short-range, high-data-rate applications recently. It is an alternative range, and many applications operate within this range, such as wireless uncompressed HD streaming, Wi-Fi, fast transfers of huge files, high-resolution radars, and personal networks $[5,6]$.

The tuneable antenna is designed in such a way that resonant frequency, operational bandwidth, radiation pattern and/or polarization can be changed manually or automatically (via software) to adapt to different services, system requirements and the environment. These procedures are generally performed using micro or nano electromechanical systems, microcontrollers, electrical Radio Frequency (RF) switches such as metal semiconductor field effect transistors, diode-based technologies such as P-type N-type insulator diodes, varactors or tuneable materials [7]. Alternatively, graphene's tuneable surface impedance can also be put to use in reconfigurable antennas. Graphene, a combined carbon sheet arranged in a hexagonal lattice in two dimensionals (2Ds), allows multifunctionality in terms of signal emission, transmission, modulation, and detection, and features broadband, high speed, compact size, and particularly low loss [8, 9].

Graphene material can be used in a tuneable antenna in such a manner that the conductivity of the surface is changed by applying an external electrical field to the graphene sheet. The graphene admittance is adjusted by applying a Direct Current (DC) voltage bias between the graphene sheet and the ground in such a way that the ON and OFF states become high and low impedance, respectively, as illustrated in Figure 1. This effect is used to vary the electrical dimension of the antennas and thus change the resonant frequency as desired. However, graphene is not limited to being an alternative to a conventional RF switch; it can also be used to make the radiating antenna itself. Graphene tuneable antennas have been mostly studied in the infrared and terahertz frequencies, as graphene can significantly reduce the size of the antenna and provide high antenna reconfigurability $[10,11]$.

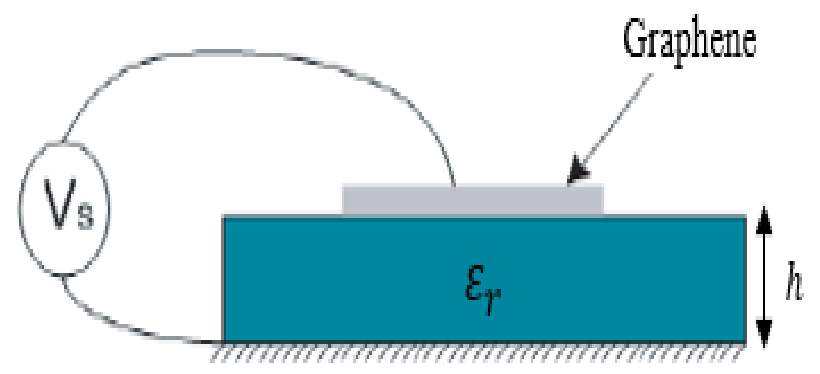

Figure 1. Graphene-based tuneable antenna

In this work, the proposed design of the tuneable single rectangular Microstrip Patch Antenna (MPA) and tuneable rectangular Microstrip Patch Array Antenna (MPAA) provides a sufficient reconfigurability range to compensate for the narrow bandwidth of Microstrip Patch Antennas (MPAs). Since mm-wave antennas made entirely of graphene are expected to have low antenna efficiencies and small reconfigurability ranges, the proposed design of the tuneable single rectangular MPA and rectangular MPAA here uses a hybrid of copper and graphene to achieve reasonable antenna efficiency and antenna gain and an acceptable tuneable frequency range. The organization of the paper can be summarized as follows: Section 2 provides a brief description of the rectangular MPA structure. Section 3 illustrates the simulation of the antenna by using the MATLAB software to obtain antenna dimensions and the Computer Simulation Technology (CST) Microwave Studio (MWS) to simulate a single rectangular MPA. Section 4 illustrates the simulation and modeling procedure of the proposed design of graphene-based tuneable single rectangular MPA. Section 5 illustrates the simulation procedure of the proposed design of graphene-based tuneable rectangular MPAA. Section 6 illustrates the discussion of the obtained results from the time domain solver of the CST-MWS after the completion of the design of the tuneable single rectangular MPA and the tuneable rectangular MPAA. Finally, Section 7 illustrates concludes of this paper.

\section{RECTANGULAR MPA}

The basic construction of the single rectangular MPA is illustrated in Figure 2. The rectangular MPA consists of a radiating patch with length $(L)$, width $(W)$, transmission line, and ground plane where are typically made from copper. The radiating patch and the transmission line sit on top of a substrate with thickness $(h)$ and permittivity $\left(\varepsilon_{r}\right)[12]$. 


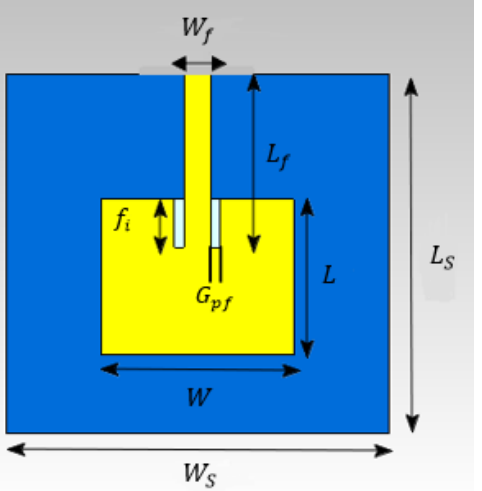

Figure 2. Dimensions of the single rectangular MPA and feed line

\section{ANTENNA DESIGN AND MODELING}

In this paper, a tuneable single rectangular MPA and rectangular MPAA based on hybrid of copper and graphene material are simulated and optimized for 5G applications. Table 1 sets the essential parameters for designing a single rectangular MPA. In this work, the inset feeding technique is used to obtain the best impedance between the antenna and the source of the electrical wave, where the input impedance of the rectangular MPA inset-feed depends on the feed inset $\left(f_{i}\right)$ and, to some extent, the gap feed line spacing $\left(G_{p f}\right)$. The resonant frequency shifts with the variation in $G_{p f}$, and the return loss of the antenna changes with the variation in $f_{i}$ [13]. The ground plane, substrate, patch, and feed line dimensions of the simulated antenna are summarized in Table 2, which has been produced with the help of MATLAB software and the following equations $[14,15]$.

Table 1. Essential antenna parameters

\begin{tabular}{ll}
\hline Parameter & Value \\
\hline Operating frequency $\left(f_{r}\right)$ & $60 \mathrm{GHz}$ \\
Substrate dielectric constant $\left(\varepsilon_{r}\right)$ & 2.1 \\
Substrate height $(h)$ & $0.1 \mathrm{~mm}$ \\
Input impedance $R_{\text {in }}$ & $50 \Omega$ \\
\hline
\end{tabular}

$$
\begin{aligned}
& W=\frac{c}{2 f_{r} \sqrt{\frac{\left(\varepsilon_{r}+1\right)}{2}}} \\
& \varepsilon_{\text {reff }}=\frac{\varepsilon_{r}+1}{2}+\frac{\varepsilon_{r}-1}{2}\left[1+12 \frac{h}{W}\right]^{\frac{-1}{2}} \\
& \Delta L=0.412 h \frac{\left(\varepsilon_{r e f f}+0.3\right)\left[\frac{W}{h}+0.264\right]}{\left(\varepsilon_{r e f f}-0.258\right)\left[\frac{W}{h}+0.8\right]} \\
& L_{e f f}=\frac{c}{2 f_{r} \sqrt{\varepsilon_{r e f f}}} \\
& L=L_{e f f}-2 \Delta L \\
& f_{i}=\frac{\cos ^{-1}\left(\sqrt{\frac{Z_{o}}{R_{\text {in }}}}\right)}{\frac{\pi}{L}} \\
& B=\frac{377 \pi}{2 Z_{0} \sqrt{\varepsilon_{r}}} \\
& W_{f}=\frac{2 h}{\pi}\left\{B-1-\ln (2 B-1)+\frac{\varepsilon_{r}-1}{2 \varepsilon_{r}}\left[\ln (B-1)+0.39-\left(\frac{0.61}{\varepsilon_{r}}\right)\right]\right\} \\
& L_{f}=3.96 \times W_{f}
\end{aligned}
$$




$$
\begin{aligned}
& G_{p f}=\frac{c \times 4.65 \times 10^{-9}}{f_{r} \sqrt{2 \varepsilon_{r e f f}}} \\
& W_{s}=2 \times W \\
& L_{s}=2 \times L,
\end{aligned}
$$

Where $\varepsilon_{\text {reff }}$ is the effective dielectric constant, $\varepsilon_{r}$ is the dielectric constant of the substrate, $h$ is the height of the dielectric substrate, $W$ is the width of the patch, $L_{e f f}$ is the effective length, $L$ is the actual patch length, $\Delta L$ is the length's extension, $Z_{o}$ is equivalent to the feed line impedance, $R_{\text {in }}$ is the resonant input resistance when the patch is fed at the radiating edge, $W_{f}$ is the feed line width, $L_{f}$ is the feed line length, $W_{s}$ is the width of the substrate, and $L_{s}$ is the length of the substrate.

Table 2. Calculated antenna parameters

\begin{tabular}{ll}
\hline Parameter & Value $(\mathrm{mm})$ \\
\hline Patch width $(W)$ & 2.008 \\
Patch length $(L)$ & 1.6681 \\
Substrate width $\left(W_{S}\right)$ & 4.016 \\
Substrate length $\left(L_{S}\right)$ & 3.3362 \\
Feed line inset $\left(f_{i}\right)$ & 0.58217 \\
Feed line width $\left(W_{f}\right)$ & 0.308 \\
Feed line length $\left(L_{f}\right)$ & 1.21968 \\
Patch feed line spacing $\left(G_{p f}\right)$ & 0.0179 \\
\hline
\end{tabular}

Once the parameter calculations are completed via the previous equations, the antenna simulation process is started via the CST-MWS. Figure 3 illustrates the single rectangular MPA inside the CST-MWS. In this work, the main aims of the optimization process are to enhance the overall antenna performance and facilitates the antenna manufacturing process (the laser machine accuracy in manufacturing antennas is $0.08 \mathrm{~mm} \mathrm{[16]).} \mathrm{Therefore,} \mathrm{the} \mathrm{optimization} \mathrm{procedures} \mathrm{for} \mathrm{the} \mathrm{antenna} \mathrm{involve} \mathrm{maximizing} \mathrm{some} \mathrm{parameters}$ and minimize some others by trial and error until the best results are reached. Table 3 summarizes the optimized parameters for a single rectangular MPA.

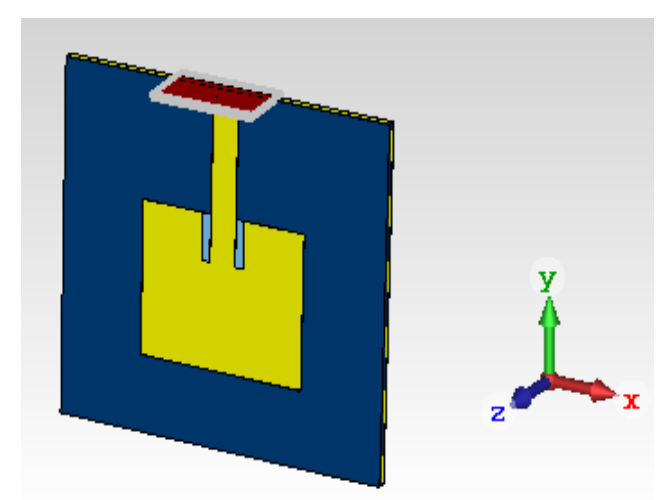

Figure 3. Simulated single rectangular MPA inside the CST-MWS

Table 3. Parameter values achieved through the optimization process

\begin{tabular}{ll}
\hline Parameter & Optimized Value $(\mathrm{mm})$ \\
\hline Patch width $(W)$ & 2 \\
Patch length $(L)$ & 1.615 \\
Substrate width $\left(W_{S}\right)$ & 4 \\
Substrate length $\left(L_{S}\right)$ & 3.23 \\
Feed line inset $\left(f_{i}\right)$ & 0.5 \\
Feed line width $\left(W_{f}\right)$ & 0.09 \\
Feed line length $\left(L_{f}\right)$ & 1.8 \\
Patch feed line spacing $\left(G_{p f}\right)$ & 0.09 \\
\hline
\end{tabular}




\section{GRAPHENE-BASED TUNEABLE SINGLE RECTANGULAR MPA}

In order to demonstrate the use of graphene in a frequency reconfigurable antenna, the main design studied is a rectangular hybrid MPA with a copper radiating patch with four graphene strips inserted inside it. The first step of the design process is to choose the graphene strips' locations within the antenna's radiating patch by monitoring the surface current distribution and select the locations of the reasonable current density. The current density at the resonant frequency shows a greater amount of accumulated current on the radiating patch, as illustrated in Figure 4.

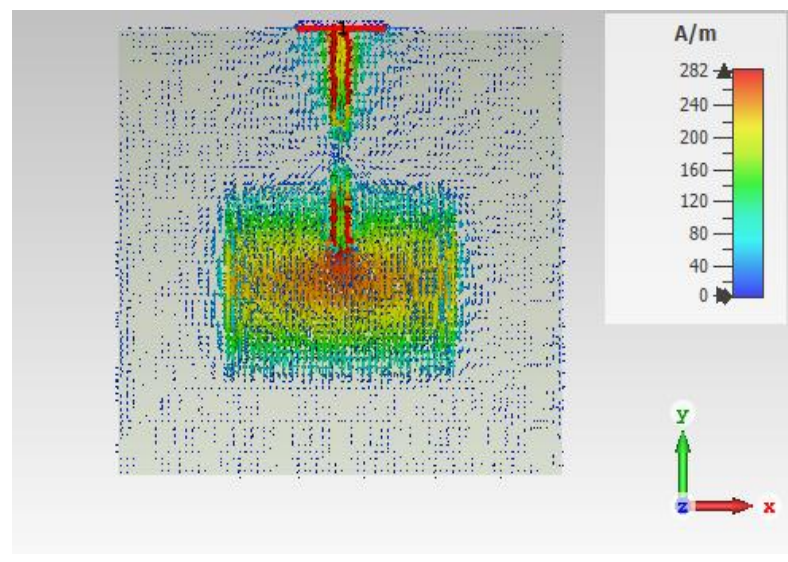

Figure 4. The surface current of the antenna at the resonance frequency

The graphene strips act as a control switch to divert the current flow along with the radiating patch of the antenna so that the effective length is larger or shorter than the physical length in the OFF or ON state, respectively. The ON state signifies low graphene impedance (i.e. allows the current to flow through the strip), and the OFF state signifies high graphene impedance (i.e. prevents the flow of the current through the strip). The DC bias voltage $\left(V_{B}\right)$ can be applied directly through the feed port if the same value of $V_{B}$ is desired for all graphene strips, allowing them all to be activated/deactivated at the same time. Alternatively, a thin gap can be inserted between the radiating patch and the graphene strips to avoid a common DC bias voltage across the antenna and allows each graphene strip to be activated and deactivated individually. As a result, different DC bias voltages (i.e. $V_{B 1}$ and $V_{B 2}$ ) can be applied to each of the graphene strips using individual DC bias lines [17]. In this work, the graphene strips are surrounded by copper. Hence, that separate coaxial ports are required to apply a different value of $V_{B}$ to each graphene strip. Here, the first approach in the antenna design is to use same $V_{B}$ for all graphene strips. In order to achieve practical values for the ON and OFF surface impedances $\left(Z_{S_{O N}}\right.$ and $\left.Z_{S_{O F F}}\right)$, the following equations are used [18-20].

$$
\begin{aligned}
& n=\frac{\varepsilon_{o} \varepsilon_{r} V_{b}}{d q} \\
& \mu_{c}=\hbar v_{f} \sqrt{n \pi} \\
& \tau_{s}=\frac{4 \hbar^{2} \rho_{m} v_{p h}{ }^{2} v_{f}}{\sqrt{n \pi} k_{B} T D^{2}} \\
& \tau_{l}=\frac{\mu_{l} \hbar \sqrt{n \pi}}{q v_{f}} \\
& Z_{s}=\frac{j \pi \hbar^{2}\left(2 \pi f_{r}\left(\tau_{l} \tau_{S}\right)-j\left(\tau_{l}+\tau_{S}\right)\right)}{q^{2}\left(\tau_{l} \tau_{s}\right)\left[\mu_{c}+2 k_{B} T \ln \left(e^{-\frac{\mu_{C}}{k_{B} T}+1}\right)\right]},
\end{aligned}
$$

Where $\tau_{l}$ is the scattering effect, $\tau_{s}$ is the relaxation time, $\mu_{l}$ represents the electron mobility $\left(\mathrm{m}^{2} / V_{s}\right), \rho_{m}=7.6 \times 10^{-7}$ is the $2 \mathrm{D}$ mass density of graphene in $\left(\mathrm{Kg} / \mathrm{m}^{2}\right), v_{p h}=2.1 \times 10^{4}$ is the sound velocity of longitudinal acoustic phonons in graphene $(\mathrm{m} / \mathrm{s}), T$ is the temperature in degrees Kelvin, $\hbar$ is the reduced Planck's constant, $k_{B}$ is the Boltzmann constant, $q$ is the electron charge $(\mathrm{C}), \mu_{c}$ is the graphene 
chemical potential $(\mathrm{Ev}), v_{f}=1 \times 10^{6}$ is the Fermi velocity $(\mathrm{m} / \mathrm{s}), n$ is the carrier density $\left(\mathrm{m}^{-2}\right), d$ is the graphene thickness $(\mathrm{m})$, and $D$ is the deformation potential $(\mathrm{eV})$. Based on the previous studies, a $D$ of around $\sim 18 \mathrm{eV}$ appears to be a prevalent and recognized value for graphene over a substrate [17]. The value of the $Z_{S_{O N}} / Z_{S_{O F F}}$ are determined by increasing/decreasing the density of the charge carriers in (16). Table 4 summarizes the selected parameter values of the graphene surface impedance in both ON and OFF states.

In this work, different values of the applied $V_{B}$ are investigated to study the effects of increasing/decreasing the $V_{B}$ on the tuning of the resonance operating frequency band. While the resulting $V_{B}$ values may be too large to be implemented in low-power applications, such as mobile phones, future values can be decreased by using a thin dielectric with greater $\varepsilon_{r}$, depending on the required application and operating frequency band. Graphene strips are simulated via CST-MWS as ohmic sheet surface impedances. Figure 5 illustrates the simulated antenna inside the CST-MWS, and Table 5 summarizes the graphene strip dimensions.

Table 4. Selected parameter values for the graphene surface impedance

\begin{tabular}{|c|c|c|c|c|c|c|}
\hline Parameter & \multicolumn{6}{|c|}{ Value } \\
\hline State & & & $\mathrm{ON}$ & & & OFF \\
\hline$\mu_{l}\left(\mathrm{~m}^{2} / \mathrm{Vs}\right)$ & & & 2.7 & & & 2.7 \\
\hline$D(\mathrm{eV})$ & & & 4 & & & 4 \\
\hline $\mathrm{T}(\mathrm{K})$ & & & 295 & & & 295 \\
\hline$V_{B}(\mathrm{~V})$ & 1 & 5 & 20 & 30 & 40 & 0.5 \\
\hline$n\left(\mathrm{~m}^{-2}\right)$ & $1.16 \times 10^{16}$ & $5.8 \times 10^{16}$ & $2.32 \times 10^{17}$ & $3.48 \times 10^{17}$ & $4.64 \times 10^{17}$ & $6 \times 10^{14}$ \\
\hline$Z_{S}(\Omega / \quad)$ & $200+25.5 \mathrm{i}$ & $27.72+9.256 \mathrm{i}$ & $11.41+5.7 \mathrm{i}$ & $8+4.65 i$ & $6.43+4 i$ & $2569.6+74.7 \mathrm{i}$ \\
\hline
\end{tabular}
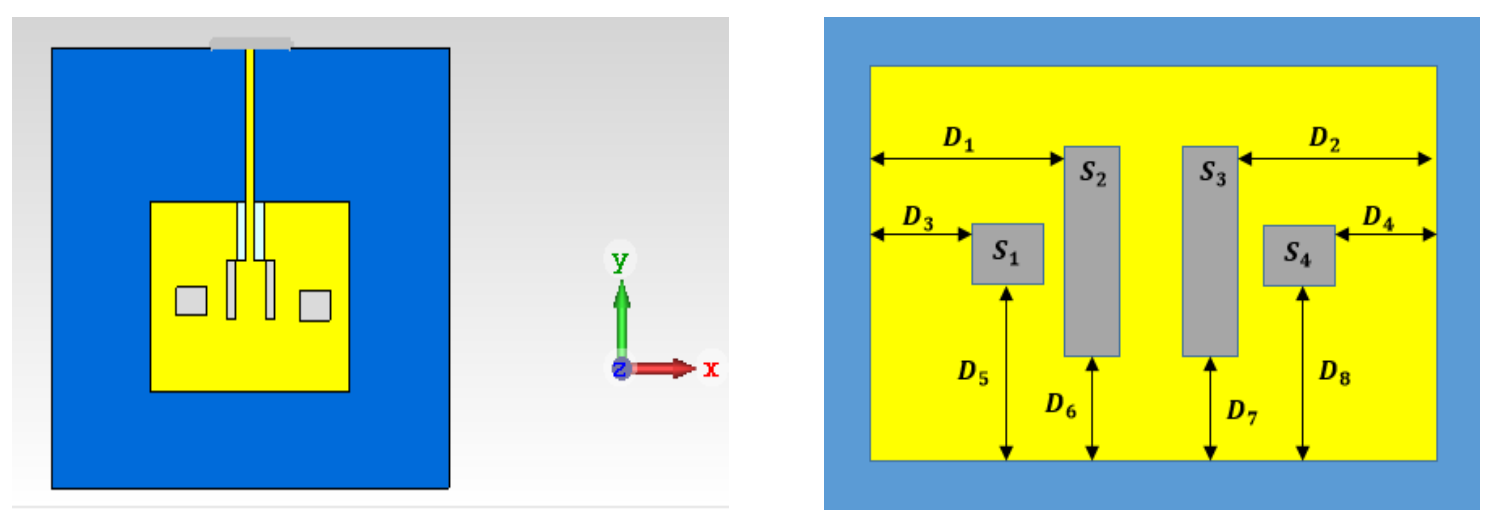

Figure 5. Simulated hybrid graphene and copper tuneable single rectangular MPA

Table 5. Graphene strip dimensions

\begin{tabular}{cccc}
\hline Strip & Dimensions & Strip & Dimensions \\
\hline$S_{1}$ & $0.24 \times 0.3 \mathrm{~mm}$ & $S_{2}$ & $0.5 \times 0.09 \mathrm{~mm}$ \\
$S_{3}$ & $0.5 \times 0.09 \mathrm{~mm}$ & $S_{4}$ & $0.25 \times 0.3 \mathrm{~mm}$ \\
$D_{1}$ & $0.26 \mathrm{~mm}$ & $D_{2}$ & $0.76 \mathrm{~mm}$ \\
$D_{3}$ & $0.26 \mathrm{~mm}$ & $D_{4}$ & $0.20 \mathrm{~mm}$ \\
$D_{5}$ & $0.65 \mathrm{~mm}$ & $D_{6}$ & $0.61 \mathrm{~mm}$ \\
$D_{7}$ & $0.61 \mathrm{~mm}$ & $D_{8}$ & $0.60 \mathrm{~mm}$ \\
\hline
\end{tabular}

\section{GRAPHENE-BASED TUNEABLE RECTANGULAR MICROSTRIP PATCH ARRAY ANTENNA}

Rectangular MPAs can be used in the form of a single antenna configuration or in a configuration of array elements. By using antenna arrays in communication systems, the antenna performance will be enhanced in terms of, for instance, gain, directivity and other functions that are difficult to achieve with a single rectangular MPA [21,22]. The simulated array is fed via a corporate feed to obtain the best performance, as illustrated in Figure 6. This technique exerts more control over the feed for each element and is perfect for scanning phased and multiband arrays. Therefore, it improves directivity as well as radiation efficiency and reduces beam variations over the frequency band compared to the series feed array. In this work, a $4 \times 1$ array antenna is used to enhance the tuneable antenna gain, directivity, efficiency and other 
parameters. The antenna element spacing plays a key role in the design process. Too large a spacing results in the presence of grating lobes that are, in most cases, undesirable. In contrast, too small a spacing leads to a wider beam width, which may not be acceptable. Smaller spacing also reduces the amount of space on the feed network. The space between the elements should, therefore, be adjusted correctly, i.e. the element spacing should be set to $0.5 \lambda$ in order to eliminate the impact of adjacent components [23]. Table 6 summarizes the corporate feed parameters before and after the optimization process. After the completion of the corporate feed design, the previous design of the tuneable single rectangular MPA is directly coupled with the corporate feed configuration to construct the tuneable rectangular MPAA, as illustrated in Figure 7.

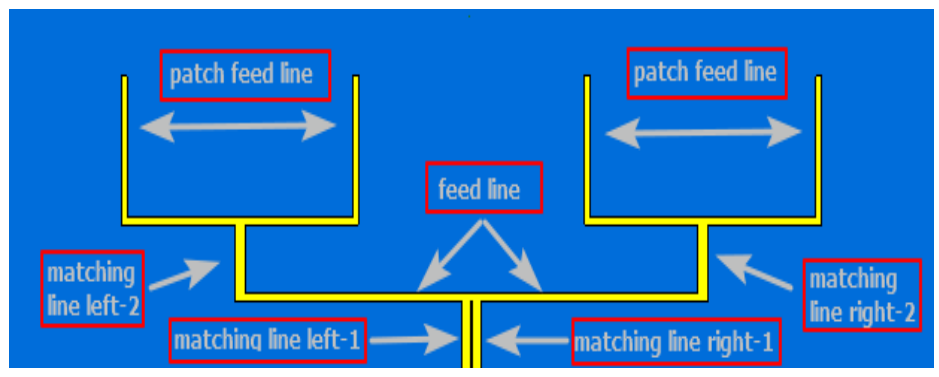

Figure 6. Corporate feed for a $4 \times 1$ rectangular MPAA

Table 6. Corporate feed parameters before and after the optimization process

\begin{tabular}{ccc}
\hline Parameter & Calculated & Optimized \\
\hline Patch feed line width & $0.1 \mathrm{~mm}$ & $0.1 \mathrm{~mm}$ \\
Patch feed line length & $1.84 \mathrm{~mm}$ & $1.72 \mathrm{~mm}$ \\
Matching line left-1 width & $0.17 \mathrm{~mm}$ & $0.16 \mathrm{~mm}$ \\
Matching line left-1 length & $0.93 \mathrm{~mm}$ & $0.91 \mathrm{~mm}$ \\
Matching line left-2 width & $0.19 \mathrm{~mm}$ & $0.18 \mathrm{~mm}$ \\
Matching line left-2 length & $0.94 \mathrm{~mm}$ & $0.92 \mathrm{~mm}$ \\
Matching line right-1 width & $0.17 \mathrm{~mm}$ & $0.16 \mathrm{~mm}$ \\
Matching line right-1 length & $0.93 \mathrm{~mm}$ & $0.91 \mathrm{~mm}$ \\
Matching line right-2 width & $0.19 \mathrm{~mm}$ & $0.18 \mathrm{~mm}$ \\
Matching line right-2 length & $0.94 \mathrm{~mm}$ & $0.92 \mathrm{~mm}$ \\
Feed line width & $0.1 \mathrm{~mm}$ & $0.1 \mathrm{~mm}$ \\
Feed line length & $8.19 \mathrm{~mm}$ & $8.18 \mathrm{~mm}$ \\
\hline
\end{tabular}

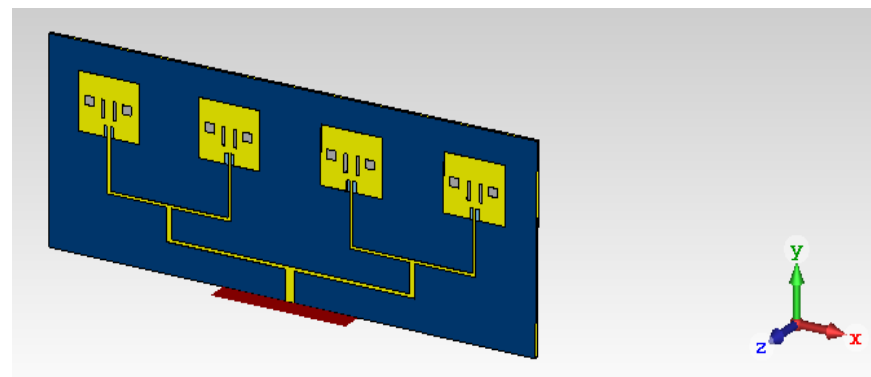

Figure 7. $4 \times 1$ tuneable rectangular MPAA inside the CST-MWS

\section{RESULTS AND DISCUSSION}

In this part, the results obtained after the antenna simulation is completed within the CST-MWS simulation software will be illustrated. The simulated return loss $\left(S_{11}\right)$ of the single rectangular MPA in the ON/OFF states from the time domain solver of the CST-MWS is illustrated in Figure 8. Generally, the MPAs have poor gain due to the antenna gain being affected by the substrate thickness and relative dielectric constant. The antenna gain is inversely proportional to $\varepsilon_{r}$ and directly proportional to substrate thickness [24]. Figure 9 shows the Three-Dimensional (3D) results of the far field of the proposed tuneable single rectangular MPA at the operating frequencies $f_{r}=56.329,56.724,59.043,59.743,59.901$ and $60 \mathrm{GHz}$. The proposed antenna has a reasonable gain in the frequency range $56.3295-60 \mathrm{GHz}$. 
The radiation pattern of the single rectangular MPA is characterized by a single main lobe of moderate beam width. Often, the beam width in the azimuth and elevation planes are similar, resulting in a fairly circular beam, although this is by no means universal [25]. The beam widths can be manipulated to produce an antenna with higher or lower gain, depending on the required application. The $3 \mathrm{~dB}$ beam widths of the proposed antenna at different previous resonance frequencies are illustrated in Figure 10.

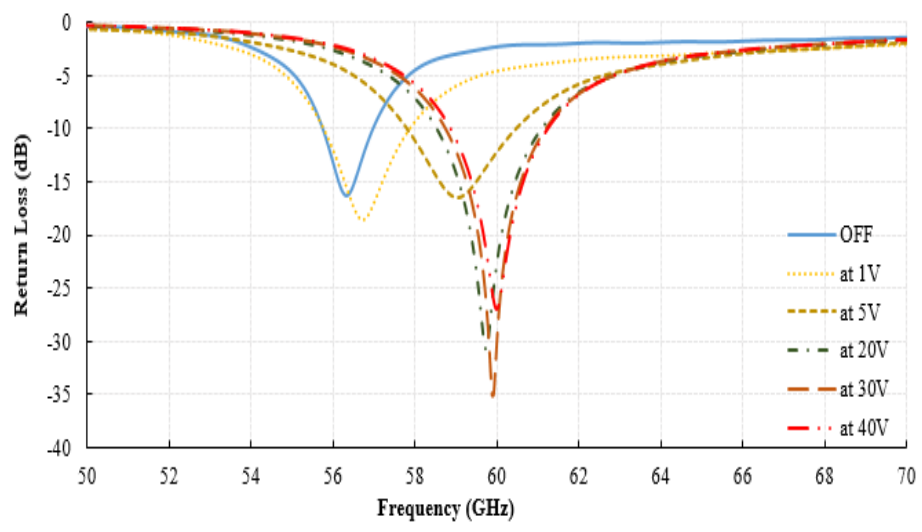

Figure 8. Return loss vs frequency of the tuneable single rectangular MPA for different values of $V_{B}$
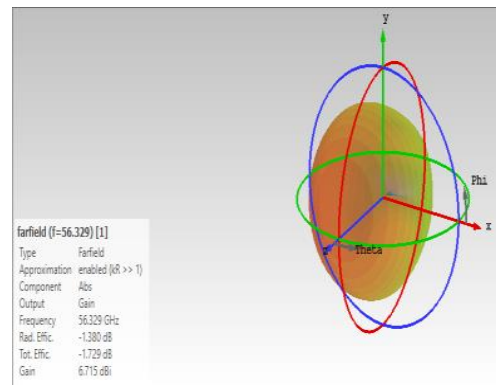

(a)

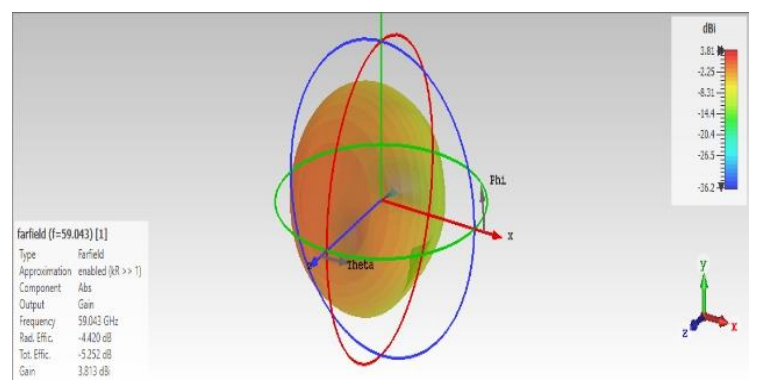

(c)

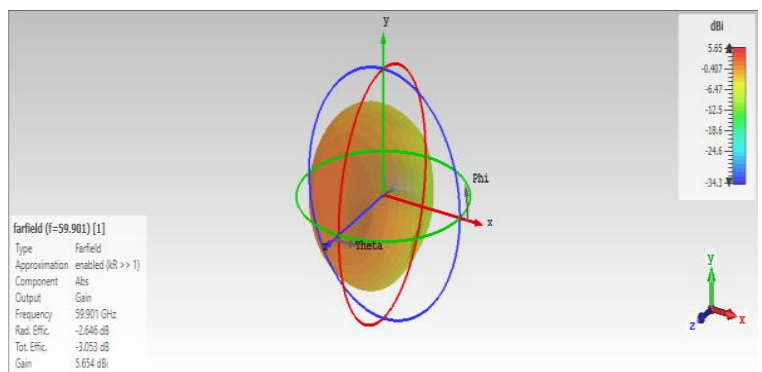

(e)

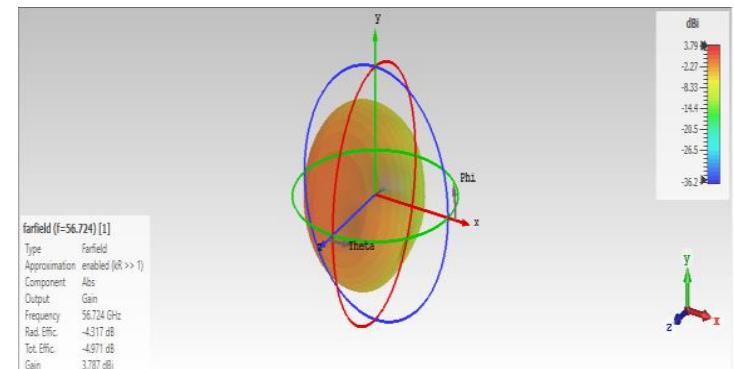

(b)

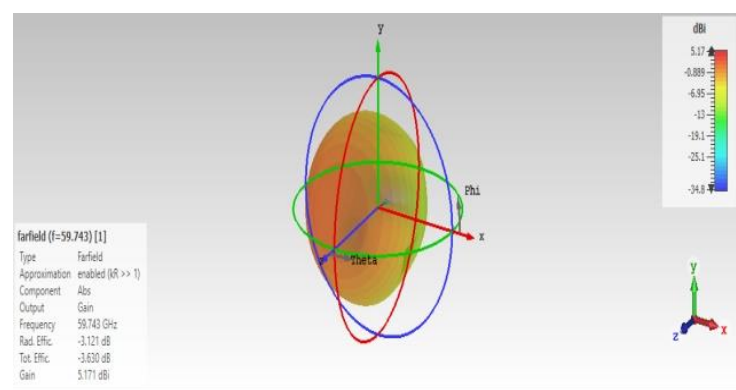

d)

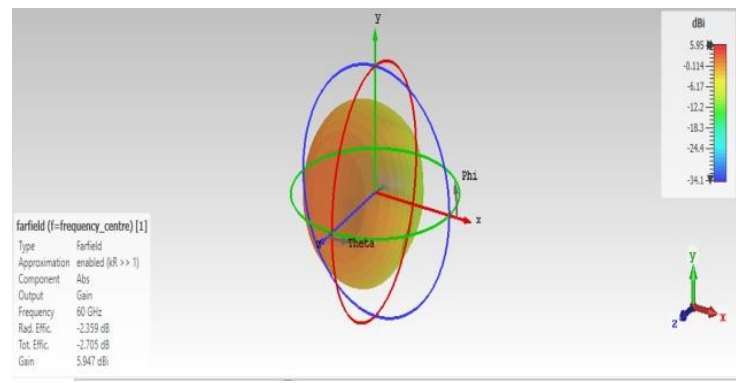

(f)

Figure 9. Gain of the tuneable single rectangular MPA, (a) $f_{r}=56.329 \mathrm{GHz}$, (b) $f_{r}=56.724 \mathrm{GHz}$, (c) $f_{r}=59.043 \mathrm{GHz}$, (d) $f_{r}=59.743 \mathrm{GHz}$, (e) $f_{r}=59.901 \mathrm{GHz}$, (f) $f_{r}=60 \mathrm{GHz}$ 


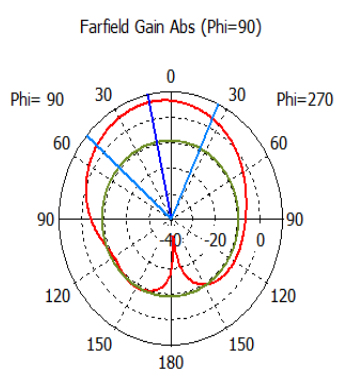

Theta / Degree vs. dBi

Farfield Gain Abs (Phi=90)

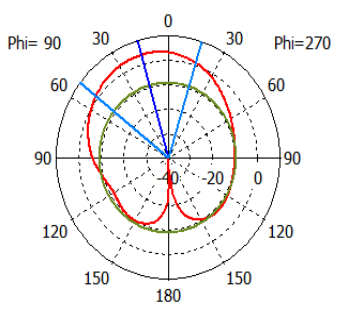

Theta / Degree vs. dBi

(c)

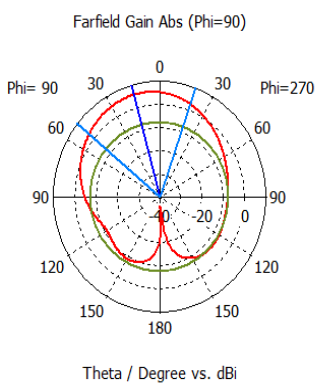

(e)

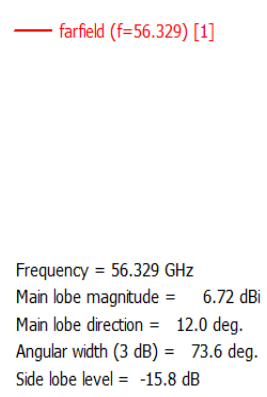

(a)

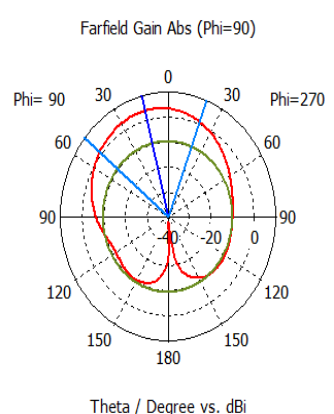

— farfield ( $f=59.043)$ [1]

Frequency $=59.043 \mathrm{GHz}$ Main lobe magnitude $=3.81 \mathrm{~dB}$ Main lobe direction $=16.0 \mathrm{deg}$. Angular width $(3 \mathrm{~dB})=69.5 \mathrm{deg}$. Side lobe level $=-12.9 \mathrm{~dB}$

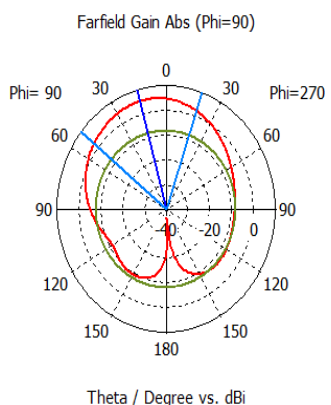

(d)

Frequency $=59.743 \mathrm{GHz}$ Main lobe magnitude $=5.17 \mathrm{~dB}$ Main lobe direction $=15.0 \mathrm{deg}$. Angular width $(3 \mathrm{~dB})=70.1 \mathrm{deg}$ Side lobe level $=-13.1 \mathrm{~dB}$

Main lobe magnitude $=3.79 \mathrm{~dB}$ Main lobe direction $=14.0$ deg. Angular width $(3 \mathrm{~dB})=71.7 \mathrm{deg}$. Side lobe level $=-13.3 \mathrm{~dB}$
Frequency $=59.901 \mathrm{GHz}$ Main lobe magnitude $=5.65 \mathrm{~dB}$ Main lobe direction $=15.0 \mathrm{deg}$. Angular width $(3 \mathrm{~dB})=70.4 \mathrm{deg}$. Side lobe level $=-13.2 \mathrm{~dB}$

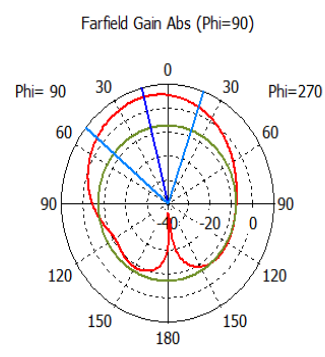

Theta / Degree vs. dBi
- farfield ( $\mathrm{f}=$ frequency centr...

Frequency $=60 \mathrm{GHz}$ Main lobe magnitude $=5.95 \mathrm{~dB}$ Main lobe direction $=14.0 \mathrm{deg}$. Angular width $(3 \mathrm{~dB})=70.5 \mathrm{deg}$. Side lobe level $=-13.3 \mathrm{~dB}$

(f)

Figure 10. Radiation pattern of the tuneable single rectangular MPA, (c) $f_{r}=59.043 \mathrm{GHz}$, (d) $f_{r}=59.743$ $\mathrm{GHz},(\mathrm{e}) f_{r}=59.901 \mathrm{GHz}$, (f) $f_{r}=60 \mathrm{GHz}$

The bandwidth of the antenna can be calculated from the return loss plot, the simulation results for the proposed tuneable single rectangular MPA bandwidth at $S_{11}=-10 \mathrm{~dB}$ are summarized in Table 7 . The simulated return losses of the tuneable rectangular MPAA for the ON/OFF states as obtained from the time-domain solver of the CST-MWS are illustrated in Figure 11. The surface current distribution of the rectangular MPAA design differs from the surface current distribution of the single rectangular MPA design, so the position of the operating frequency is shifted slightly toward the lower $f_{r}$. The 3D results of the gain of the $4 \times 1$ tuneable rectangular MPAA at the operating frequencies $f_{r}=55.94,56.43,58.917$, $59.647,59.8295$ and $59.9515 \mathrm{GHz}$ are illustrated in Figure 12. In order to provide a comparison of the tuneable single rectangular MPA and the tuneable rectangular MPAA, the gain have been enhanced.

Table 7. Bandwidth results for the tuneable single rectangular MPA

\begin{tabular}{cc}
\hline $\begin{array}{c}\text { Operating Frequency } \\
(\mathrm{GHz})\end{array}$ & $\begin{array}{c}\text { Bandwidth } \\
(\mathrm{GHz})\end{array}$ \\
\hline 56.329 & 1.2373 \\
56.724 & 2.1185 \\
59.043 & 2.6414 \\
59.743 & 2.6262 \\
59.901 & 2.5029 \\
60 & 2.4051 \\
\hline
\end{tabular}


Generally, antennas with wide beam widths have a low gain, while antennas with narrow beam widths lead to higher gain; therefore, an antenna that directs most of its energy into a narrow beam will have a higher gain. When using the $4 \times 1$ tuneable rectangular MPAA, the $3 \mathrm{~dB}$ beam width is narrower, as illustrated in Figure 13. It is observed from Figure 9 and Figure 12; that the gain of the previous designs is enhanced. Table 8 summarizes the results of the comparison of the tuneable single rectangular MPA and the tuneable rectangular MPAA.

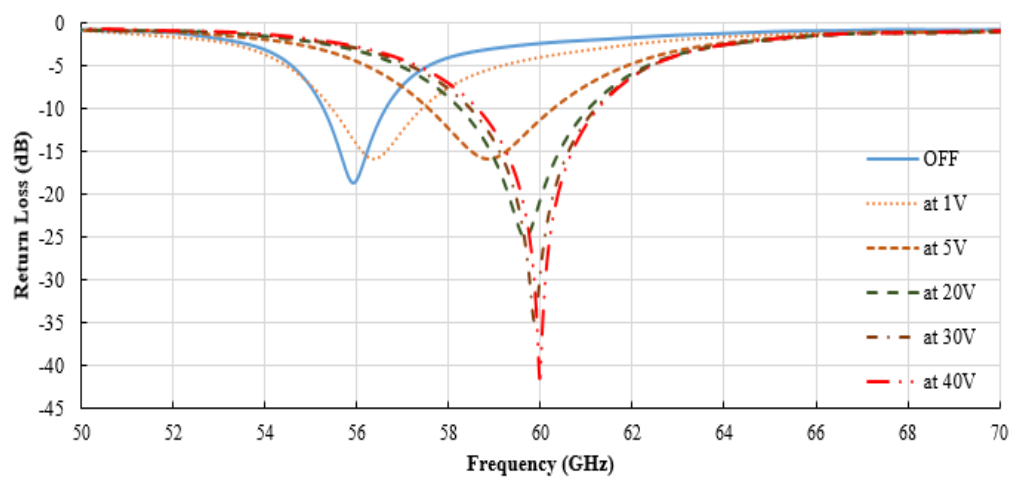

Figure 11. Return loss vs frequency of the tuneable rectangular MPAA for different values of $V_{B}$
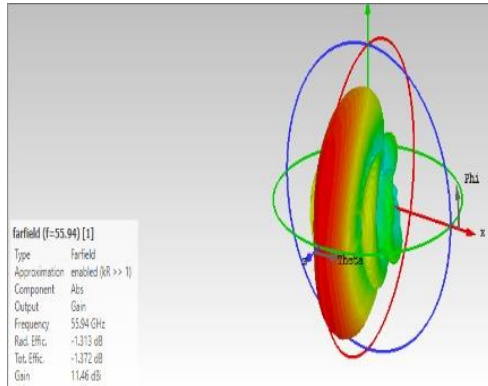

(a)

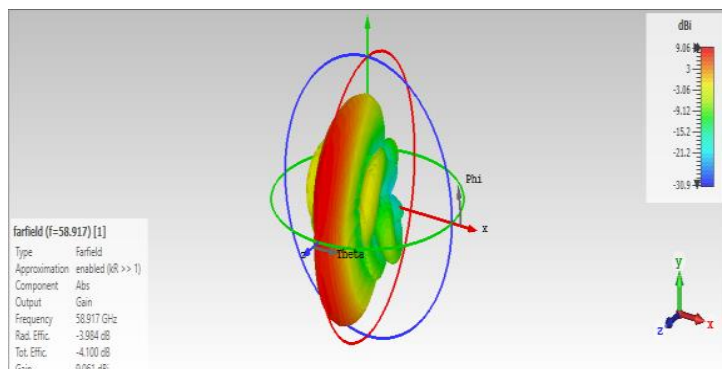

(c)

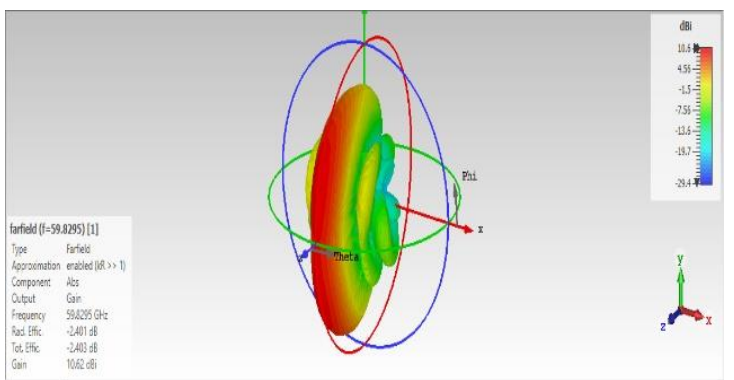

(e)

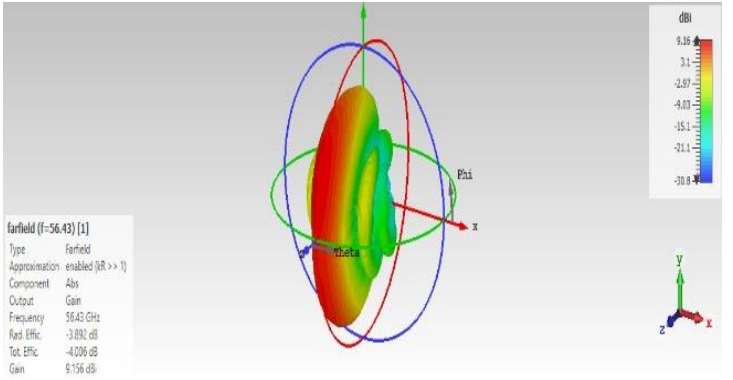

(b)

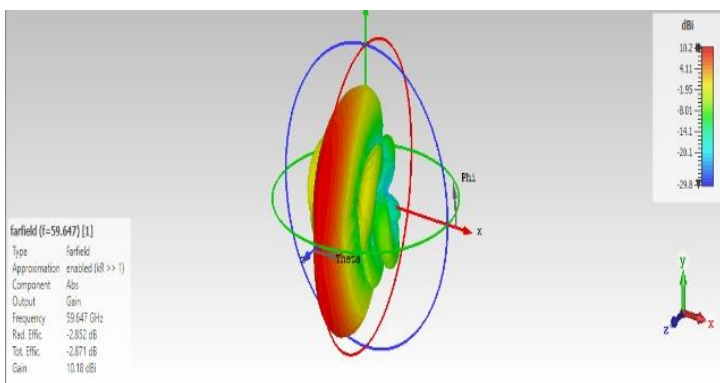

(d)

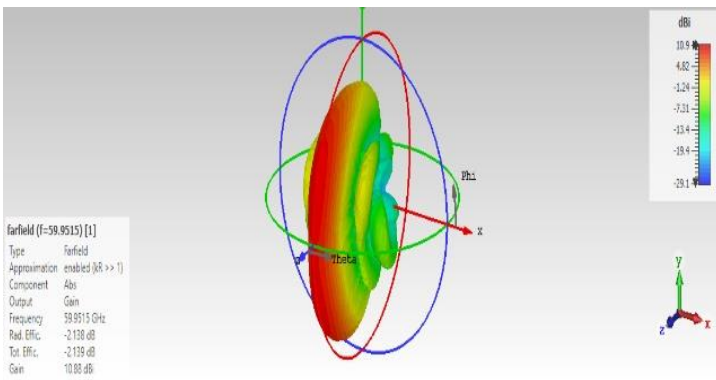

(f)

Figure 12. Gain of the tuneable rectangular MPAA, (a) $f_{r}=55.94 \mathrm{GHz}$, (b) $f_{r}=56.43 \mathrm{GHz}$, (c) $f_{r}=58.917 \mathrm{GHz}$, (d) $f_{r}=59.647 \mathrm{GHz}$, (e) $f_{r}=59.8295 \mathrm{GHz}$, (f) $f_{r}=59.9515 \mathrm{GHz}$ 


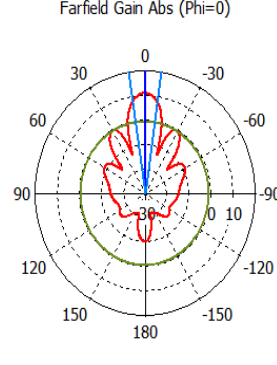

Theta / Degree vs. dB (a)

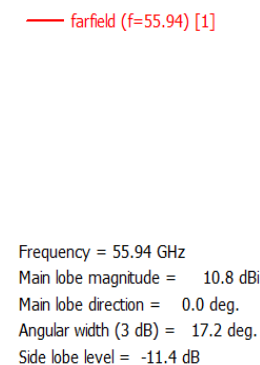

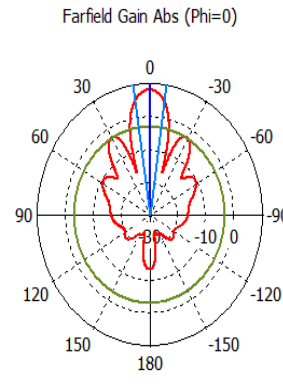

Theta / Degree vs. dBi

(b)

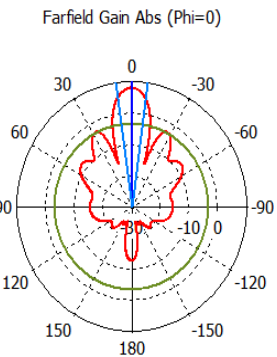

— farfield $(f=58.917)[1]$

Frequency $=58.917 \mathrm{GHz}$ Main lobe magnitude $=7.93 \mathrm{~dB}$ Main lobe direction $=0.0 \mathrm{deg}$. Angular width $(3 \mathrm{~dB})=16.2 \mathrm{deg}$. Side lobe level $=-11.1 \mathrm{~dB}$

(c)

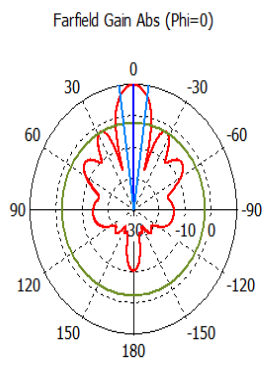

(e)

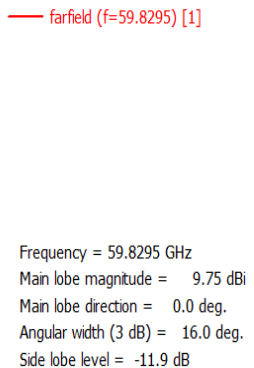

Side lobe level $=-11.9 \mathrm{~dB}$

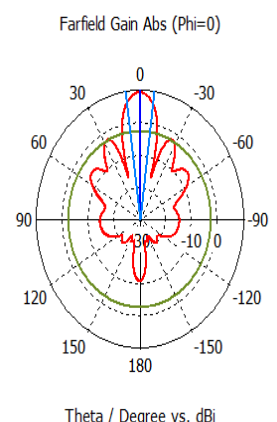

(d)

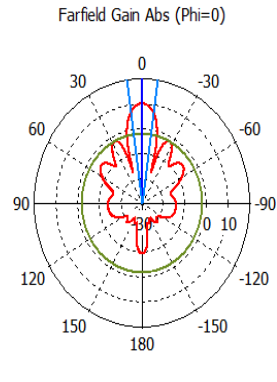

(f)
— farfield $(f=59.647)[1]$

Frequency $=59.647 \mathrm{GHz}$ Main lobe magnitude $=9.24 \mathrm{dBi}$ Main lobe direction $=0.0 \mathrm{deg}$. Angular width $(3 \mathrm{~dB})=16.0 \mathrm{deg}$ Side lobe level $=-11.7 \mathrm{~dB}$

Figure 13. Radiation patterns for the $4 \times 1$ tuneable rectangular MPAA, (a) $f_{r}=55.94 \mathrm{GHz}$, (b) $f_{r}=56.43 \mathrm{GHz}$, (c) $f_{r}=58.917 \mathrm{GHz}$, (d) $f_{r}=59.647 \mathrm{GHz}$, (e) $f_{r}=59.8295 \mathrm{GHz}$, (f) $f_{r}=59.9515 \mathrm{GHz}$

Table 8. Comparison results of the previous designs

\begin{tabular}{|c|c|c|c|c|c|c|c|c|}
\hline \multirow[b]{2}{*}{ Applied $\left(V_{B}\right)$} & \multicolumn{4}{|c|}{ Tuneable Single Rectangular MPA } & \multicolumn{4}{|c|}{ Tuneable Rectangular MPAA } \\
\hline & $\begin{array}{c}f_{r} \\
(\mathrm{GHz})\end{array}$ & $\begin{array}{l}\text { Gain } \\
(\mathrm{dBi})\end{array}$ & $\begin{array}{l}\text { Return Loss } \\
(\mathrm{dB})\end{array}$ & $\begin{array}{c}\text { Bandwidth } \\
\text { (GHz) }\end{array}$ & $\begin{array}{c}f_{r} \\
(\mathrm{GHz})\end{array}$ & $\begin{array}{l}\text { Gain } \\
(\mathrm{dBi})\end{array}$ & $\begin{array}{c}\text { Return } \\
\text { Loss }(\mathrm{dB})\end{array}$ & $\begin{array}{c}\text { Bandwidth } \\
\text { (GHz) }\end{array}$ \\
\hline 0.5 (OFF) & 56.329 & 6.71 & -16.4 & 1.2373 & 55.92 & 11.5 & -18.69 & 1.3487 \\
\hline 1 & 56.724 & 3.79 & -18.6 & 2.1185 & 56.43 & 9.16 & -15.8 & 1.9614 \\
\hline 5 & 59.043 & 3.81 & -16.5 & 2.6414 & 58.917 & 9.06 & -15.84 & 2.705 \\
\hline 20 & 59.743 & 5.17 & -29.6 & 2.6262 & 59.647 & 10.2 & -25 & 2.7766 \\
\hline 30 & 59.901 & 5.65 & -37.5 & 2.5029 & 59.829 & 10.6 & -34.8 & 2.707 \\
\hline 40 & 60 & 5.95 & -27.8 & 2.4051 & 59.951 & 10.9 & -41.5 & 2.6096 \\
\hline
\end{tabular}

\section{CONCLUSION}

In this paper, reconfigurable hybrid metal-graphene single/array rectangular MPAs have been simulated and optimized for 5G applications via the CST-MWS package. The approach used in this work involves using planted graphene strips inside a copper radiating patch to obtain a tuneable antenna with a reasonable performance. The designed antenna has a good gain and many frequencies in range of 55.75 to $61.26 \mathrm{GHz}$ in the tuneable single rectangular MPA and the same results were obtained in the array configuration but with some shifting toward the lower $f_{r}$ due to the surface current distribution of 
the rectangular MPAA design differs from the surface current distribution of the single rectangular MPA design. This depends on the voltage applied to the graphene strips. From this work, it can be concluded that increasing the applied voltage on the graphene strips leads to reducing their surface impedances, resulting a shift in the resonance frequency towards the upper frequency. Finally, the MPA bandwidth is expanded. As noted from the above-mentioned comparison results involving the tuneable single/array rectangular MPA designs, the gain has improved significantly.

\section{REFERENCES}

[1] L. Ching and M. R. Kamarudin, "Investigation of Patch Phase Array Antenna Orientation at $28 \mathrm{GHz}$ for 5G Applications," Procedia Computer Science, vol. 86, pp. 47-50, 2016.

[2] S. N. H. Sa'don, et al., "A 5G graphene antenna produced by screen printing method," Indonesian Journal of Electrical Engineering and Computer Science (IJEECS), vol. 15, no. 2, pp. 950-955, 2019.

[3] R. Wang, et al., "A Planar End-Fire Antenna with Wide Beamwidth for $60 \mathrm{GHz}$ Applications," Progress in Electromagnetics Research Letters, vol. 61, pp. 125-130, 2016.

[4] T. J. Kao, et al., "Design and Analysis of a 60-GHz CMOS Doppler and Vibration Detection," IEEE Transactions on Microwave Theory and Techniques, vol. 61, no. 4, pp. 1649-1659, 2013.

[5] A. S. W. Ghattas, et al., "Compact Simple Planar Millimeter- Wave Antenna for Unlicensed 57-64 GHz Band Applications," 2018 International Japan-Africa Conference on Electronics, Communications and Computations (JAC-ECC), Alexandria, Egypt, pp. 107-110, 2018.

[6] N. Qasem, "Enhancing the Capacity of the Indoor $60 \mathrm{GHz}$ Band Via Modified Indoor Environments Using Ring Frequency Selective Surface Wallpapers and Path Loss Models," International Journal of Electrical and Computer Engineering, vol. 8, no. 5, pp. 3003-3020, 2018.

[7] N. Qasem and H. M. Marhoon, "Simulation and optimization of a tuneable rectangular microstrip patch antenna based on hybrid metal-graphene and FSS superstrate for fifth-generation applications," TELKOMNIKA Telecommunication, Computing, Electronics and Control, vol. 18, no. 4, pp. 1719-1730, 2020.

[8] H. A. Abdulnabi and Y. Y. Al-aboosi, "Design of Tunable Multiband Hybrid Graphene Metal Antenna in Microwave Regime," Indonesian Journal of Electrical Engineering and Computer Science (IJEECS), vol. 12, no. 3, pp. 1003-1009, 2018.

[9] Nanda B. S. and Puttaswamy P. S., "Modeling and simulation of graphene field effect transistor (GFET)," International Journal of Electrical and Computer Engineering, vol. 9, no. 6, pp. 4826-4835, 2019.

[10] M. Bozzi, et al., "Applications of Graphene at Microwave Frequencies," Radio Engineering, vol. 24, no. 3, pp. 661-669, 2015.

[11] A. Hlali, Zied Houaneb, and Hassen Zairi., "Dual-Band Reconfigurable Graphene-Based Patch Antenna in Terahertz Band : Design, Analysis and Modeling Using WCIP Method," Progress in Electromagnetics Research C, vol. 87, pp. 213-226, 2018.

[12] E. Thakur, et al., "Mathematical Analysis of Commonly Used Feeding Techniques in Rectangular Microstrip Patch Antenna," Advances in Signal Processing and Communication, vol. 526, pp. 27-35, 2019.

[13] M. S. Islam, et al., "Microstrip patch antenna with defected ground structure for biomedical application," Bulletin of Electrical Engineering and Informatics (BEEI), vol. 8, no. 2, pp. 586-595, 2019.

[14] S. Elajoumi, et al., "Bandwidth enhancement of compact microstrip rectangular antennas for UWB applications," TELKOMNIKA Telecommunication, Computing, Electronics and Control, vol. 17, no. 3, pp. 1559-1568, 2019.

[15] M. D. S. Mesquita, "A New Conductive Ink for Microstrip Antenna and Bioinspired FSS Designs on Glass and Fiberglass Substrates," Journal of Microwaves, Optoelectronics and Electromagnetic Applications, vol. 18, no. 2, pp. 227-245, 2019.

[16] B. S. Taha, et al., "Simulating of RF energy harvesting micro-strip patch antenna over 2.45 GHZ," International Journal of Engineering and Technology, vol. 7, no. 4, pp. 5484-5488, 2018.

[17] C. N. Alvarez, et al., "Performance Analysis of Hybrid Metal-Graphene Frequency Reconfigurable Antennas In The Microwave Regime," in IEEE Transactions on Antennas and Propagation, vol. 65, no. 4, pp. 1558-1569, Apr. 2017.

[18] G. Samanta and D. Mitra, "Wideband THz antenna using graphene based tunable circular reactive impedance substrate," Optik, vol. 158, pp. 1080-1087, 2018.

[19] M. Qu, et al., "Design of a Graphene-based Tunable Frequency Selective Surface and Its Application for Variable Radiation Pattern of a Dipole at Terahertz," Radio Science, vol. 53, no. 2, pp. 183-189, 2018.

[20] H. A. Abdulnabi, et al., "UWB THz plasmonic microstrip antenna based on graphene," TELKOMNIKA Telecommunication, Computing, Electronics and Control, vol. 18, no. 1, pp. 30-36, 2020.

[21] E. Dheyab and N. Qasem, "Design and Optimization of Rectangular Microstrip Patch Array Antenna Using Frequency Selective Surfaces for $60 \mathrm{GHz}$," International Journal of Applied Engineering Research, vol. 11, no. 7, pp. 4679-4687, 2016.

[22] A. Al-Ahmadi and Y. S. H. Khraisat, "Bandwidth Enhancement of Microstrip Patch Antenna," Applied Physics Research, vol. 11, no. 1, pp. 35-40, 2019.

[23] S. U. Rahman, et al., "Analysis of Linear Antenna Array for minimum Side Lobe Level, Half Power Beamwidth, and Nulls control using PSO," Journal Microwaves, Optoelectronics and Electromagnetic Applications, vol. 16, no. 2, pp. 577-591, 2017. 
[24] R. G. Mishra, et al., "Analysis of the Relationship between Substrate Properties and Patch Dimensions in Rectangular-Shaped Microstrip Antennas," in Intelligent Communication, Control and Devices, pp. 65-72, 2018.

[25] R. Janarthanan and S. Vinayagapriya, "High Gain Microstrip Array Antenna for Wlan Applications," International Journal of Electrical and Electronic Engineering and Telecommunication, vol. 1, no. 1, pp. 384-389, 2015.

\section{BIOGRAPHIES OF AUTHORS}

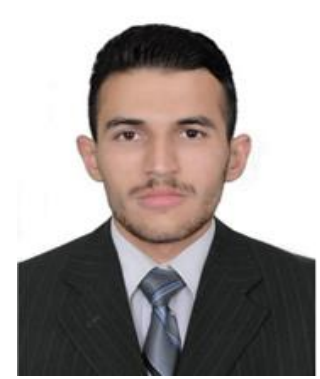

Hamzah M. Marhoon received his B.Sc. degree in Electrical Engineering (Rank 2) from College of Engineering Al-Mustansiriyah University, Baghdad, Iraq in 2016. He obtained his M.Sc. degree in Communications Engineering in 2020 from Al-Ahliyya Amman University, Hashemite Kingdom of Jordan. He works as a teaching assistant at Al-Esraa University College, Baghdad, Iraq. His research interests include Antennas, Tuneable Antennas, IoT, Energy Harvesting Antennas, and Embedded Systems.

Email: hamzaalazawy33@yahoo.com

ORCID iD: https://orcid.org/0000-0001-5613-6685

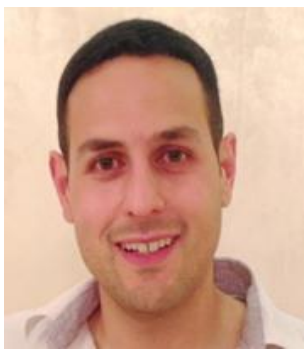

Nidal Qasem received his B.Sc. degree in Electronics and Communications Engineering (Honours) from AL-Ahliyya Amman University, Amman, Jordan, in 2004. He obtained his M.Sc. degree in Digital Communication Systems for Networks \& Mobile Applications (DSC) from Loughborough University, Loughborough, United Kingdom, in 2006. He then pursued a PhD degree in wireless and digital communication systems at Loughborough University. $\mathrm{He}$ is currently working as an associate professor in the department of Electronics and Communications Engineering at Al-Ahliyya Amman University. His research interests include propagation control in buildings, specifically improving the received power, FSS measurements and designs, antennas, ultra-wide band, orbital angular momentum, and wireless system performance analyses. He is a senior member of the IEEE. 